\title{
Visualising the risks of clinical trials for fair treatment and appropriate informed consent of participants
}

\author{
Alexander R. Harris ${ }^{1}$. Frederic Gilbert ${ }^{2}$ \\ Received: 4 January 2022 / Revised: 4 January 2022 / Accepted: 6 January 2022 / Published online: 12 January 2022 \\ (c) The Author(s), under exclusive licence to Springer-Verlag GmbH Germany, part of Springer Nature 2022
}

\section{Dear Editor,}

We'd like to thank Lindner for his response "The thin line between seeing risks and venturing scientific progress" [1] to our article "Restoring vision using optogenetics without being blind to the risks" [2]. Our article highlighted some potential risks of a clinical trial of optogenetics treatment for retinitis pigmentosa which showed significant potential after a first-in-human trial demonstrated partial recovery of visual function in a blind patient [3].

In highlighting the potential risks to first-in-human trial participants, we referenced some well-known adverse events from previous clinical trials and other risks discussed in the scientific literature or mass media. A potential immune response to the viral vector is only one risk associated with these types of trials. Lindner has correctly pointed out the difference between adeno-associated virus (AAV) and adenovirus vectors and that the current COVID-19 vaccines are in fact adenovirus vectors, and not AAVs. However, these examples were not intended to raise specific concerns about individual viral vectors but rather to demonstrate that the risks associated with these types of trials may be difficult to predict or unexpected, can have very low occurrence rates, or appear at time intervals long after initial treatment. Even when a similar therapy has successfully passed through clinical trial, a novel therapy can have a different risk profile. So while AAV2.7m8 might have shown greater

Alexander R. Harris

alexrharris@gmail.com

$\bowtie$ Frederic Gilbert

frederic.gilbert@utas.edu.au

1 Aikenhead Centre for Medical Discovery, ARC Centre of Excellence for Electromaterials Science, Faculty of Medicine, Dentistry and Health Sciences, University of Melbourne, Melbourne, Vic 3010, Australia

2 School of Humanities, University of Tasmania, Hobart, Australia transduction efficacy in preclinical studies compared to the already approved wildtype AAV2, it may yet induce unexpected side effects and not receive regulatory approval.

A main concern for these types of trials is their irreversibility, which prevents patient's right to withdraw from the trial and affects their opportunity and eligibility for future treatments [4]. From this standpoint, running irreversible clinical trials for "the sole purpose of novelty" would be unethical. For instance, a company trialling a novel AAV that aims to replicate the performance of AAV2.7m8 only to evade intellectual property rights would place a patient at risk with limited benefit. In the current optogenetics trial, AAV2.7m8 was shown to have better efficacy than the already approved wildtype AAV2. Therefore, we believe that the use of AAV2.7m8 in the clinical trial was ethically justified. However, given the knowledge already available from previous clinical trials, we question whether the trial structure used for testing AAV2.7m8 was the most appropriate for minimising participant risk and maximising trial benefit. For instance, were the number of participants, length of the trial, and viral dosage study appropriate? What support is available to the participants at the conclusion of the trial whether it was successful or not? It also raises concerns about development of newer vectors or optogenetic sequences; what amount of improvement in preclinical trial data would justify a new vector or optogenetic sequence being trialled in humans and how should that trial be structured? At what level of development of optogenetics, bionic vision devices (or other therapies) would trials of novel therapies for vision loss become unethical?

Lindner points out that COVID-19 and HIV are highly emotional topics and that raising documented adverse events from large-scale clinical trials of viral vector use may plant unnecessary concerns in potential optogenetics trial participants. However, all patients suffering from progressive, untreatable diseases are likely to be highly emotional, regardless of the disease prevalence or media coverage. And it is difficult to obtain accurate, long-term risk data from 
preclinical studies and short-term first-in-human trials from small populations. It is therefore critical to communicate the range of possible risks a trial participant may encounter, even very low potential risks, to balance their emotional state.

Trial sponsors cannot demand or expect that patients will automatically participate in a clinical trial. While risks are never fully eliminated during a clinical trial, patients must be convinced that the trial will have benefits to society, and where possible, the risks have been minimised. Information is provided to trial participants to obtain appropriate informed consent and ensure that they are treated fairly. Patients must be allowed to decide if they want to participate in a trial, receive a different therapy now or in the future, or not have any therapy. Withholding information from potential trial participants with the fear it would prevent them joining a clinical trial is unethical and belong in the realm of exploitative and abusive malpractices. It may assume that participants do not have the intelligence or agency to make their own decisions or it may deliberately be downplaying or ignoring treatment risks that should be managed or eliminated. And if a preventable adverse event subsequently does occur, it may not just impact on the participant, but may affect further development of the therapy and have consequences for those running the trial. Ultimately, if the risk-benefit ratio of a clinical trial is too high, then a participant has a right to opt out from joining, the trial could be restructured to better balance the risk-benefit ratio, or its implementation could be questioned [5].

\section{References}

1. Lindner M The thin line between seeing risks and venturing scientific progress. Graefe's Arch ClinExp Ophthalmol

2. Harris AR, Gilbert F (2021) Restoring vision using optogenetics without being blind to the risks. Graefe's Arch ClinExp Ophthalmol.https://doi.org/10.1007/s00417-021-05477-6

3. Sahel J-A, Boulanger-Scemama E, Pagot C, et al (2021) Partial recovery of visual function in a blind patient after optogenetic therapy. Nat Med.https://doi.org/10.1038/s41591-021-01351-4

4. Gilbert F Harris AR Kapsa RMI (2014) Controlling brain cells with light: ethical considerations for optogenetic clinical trials. AJOB Neurosci 5:3-11. https://doi.org/10.1080/21507740.2014. 911213

5. Harris AR, Gilbert F (2022) Military medicine research: incorporation of high risk of irreversible harms into a stratified risk framework for clinical trials. In: Messelken D., Winkler D. (eds) Health Care in Contexts of Risk, Uncertainty, and Hybridity. Military and Humanitarian Health Ethics. Springer, Cham., pp 253-273.https:// doi.org/10.1007/978-3-030-80443-5_16

Publisher's note Springer Nature remains neutral with regard to jurisdictional claims in published maps and institutional affiliations. 